\title{
Maternal and perinatal complications in triplet compared with twin pregnancy
}

\author{
Job G. Santema, Petra Bourdrez, Henk C.S. Wallenburg* \\ Department of Obstetrics and Gynecology, Erasmus University School of Medicine and Health Sciences, EE 2283, P.O. Box 1738, \\ 3000 DR Rotterdam. The Netherlands
}

Accepted 19 February 1995

\begin{abstract}
Objective: To compare maternal and perinatal complications in triplet and twin pregnancies. Study design: Case-controlled study in the setting of a University Hospital. Each pregnancy of a consecutive series of 40 triplet pregnancies of 20 weeks or more was matched for parity and maternal age with two sets of twins delivered in the same year. Primary end points of the analysis were maternal complications and perinatal outcome. Results: Of the triplets $82 \%$ and of the twins $36 \%$ were a result of assisted reproduction. Pre-term labor occurred significantly more often in triplet than in twin gestation. Triplets had a significantly lower median birth-weight (1478 vs. $2030 \mathrm{~g}$ ) and gestational age at delivery ( $32 \mathrm{vs.} 35.5$ weeks). The mean neonatal hospital stay was significantly longer in triplets, mainly related to the lower birth-weight, but there was no significant difference between triplets and twins in the incidence of major neonatal complications. Conclusion: This data of the anticipated perinatal outcome in triplet and twin pregnancies may be used to counsel women with a triplet pregnancy considering selective reduction to twins. All methods of assisted reproduction should aim at prevention of multifetal gestation.
\end{abstract}

Keywords: Triplet pregnancy; Twin pregnancy; Maternal complications; Perinatal outcome; Selective pregnancy reduction

\section{Introduction}

Ovulation induction, in vitro fertilization, and related methods of assisted reproductive technology are associated with a considerable risk of triplet and higher multiple pregnancies [1]. In an attempt to reduce the maternal and perinatal risks of multifetal pregnancy, parents are being offered the option of reduction of the number of fetuses in the first trimester of pregnancy, with the aim of improving the chances of survival and normality of the fetuses that remain [2-5]. The justification for this recommendation depends on the assessment of the balance of the risks of continuation of the triplet or higher multiple gestation, and those of selective fetocide and reduction to twin or singleton pregnancy. Pregnancies with four or more fetuses are clearly associated with an adverse perinatal outcome [2], but assumptions made with regard to the outcome of triplet pregnancy are

* Corresponding author, Tel.: 31104087596 ; Fax: 31104087532. based on relatively small series, some of which have taken 25 years to collect. Obstetric and neonatal practices have changed considerably over that period of time and may not reflect current standards of obstetric and neonatal care. In addition, very few studies report the course and outcome of triplet gestations in comparison with twin pregnancies as controls [6,7]. For that reason, we designed this study to evaluate the triplet pregnancies managed in our perinatal center from 1981-1992 and to compare their course and outcome with those of twin pregnancies in a matched-pair analysis.

\section{Materials and methods}

We reviewed the charts of all triplet pregnancies delivered after a gestational age of 20 weeks or more in the University Hospital Rotterdam-Dijkzigt (AZR-D) between January 11981 and December 31 1991, an 11 year period in which fetal reductions were not yet performed in our institution. The AZR-D is a tertiary care 
perinatal referral center serving a region with 35000 deliveries per year. Each triplet pregnancy was matched for maternal parity and age (to within 1 year) with the first two sets of twins registered in the AZR-D before or after the index case that met the matching criteria. The matching was done in a blinded fashion, without knowledge of the course and outcome of pregnancy in the control cases. Antepartum management of uncomplicated multiple pregnancy was on an outpatient basis, with standard obstetric surveillance and regular ultrasound assessment of fetal growth. Antenatal screening for fetal chromosomal abnormalities was offered on the basis of maternal age ( $\geq 36$ years) or the presence of a known risk factor. No prophylactic measures such as bed rest, cervical cerclage, or oral tocolysis were taken. Patients were admitted only because of obstetric complications.

Outcome measures were analyzed using the following definitions. In pregnancies that were a result of assisted reproduction, the calculated length of gestation was based on the date of in vitro fertilization or induced ovulation plus 2 weeks. In spontaneous pregnancies, gestational age was based on the date of the last menstrual period and confirmed by early ultrasonography. The criteria recommended by the International Society for the Study of Hypertension in Pregnancy were used to define hypertensive disorders [8]. Anemia was defined as a hemoglobin concentration of $10 \mathrm{~g} / \mathrm{dl}$ or less. Hospitalization before 37 completed weeks of gestation due to an objective or subjective increase in uterine activity requiring tocolysis, with or without associated cervical change, was considered pre-term labor. Demonstrated vaginal loss of amniotic fluid before 37 completed weeks was considered as pre-term rupture of membranes (PROM).

The diagnosis of postpartum hemorrhage was based on clinical assessment, with a blood loss of $1000 \mathrm{ml}$ or more. Birth-weight was standardized for gestational age using a birth-weight index defined as the quotient of the actual birth-weight and the birth-weight on the 50th percentile of the Dutch intrauterine growth curve for singleton pregnancies, corrected for gestational age, sex and parity [9]. Stillbirth was defined as a fetal loss with a weight of $500 \mathrm{~g}$ or more. Early neonatal mortality was defined as the number of liveborn infants weighing 500 $\mathrm{g}$ or more who died during the first 7 days after birth, and late neonatal mortality as the number of deaths of liveborn infants between 8 and 28 days after birth, per 100 liveborns. The sum of stillbirths and neonatal deaths per 100 births constitutes perinatal mortality.

Variables are presented as median and range throughout. Wilcoxon's rank sum test was used to assess continuous variables, correlations were assessed with the nonparametric Spearman test, and discrete data were analyzed with the $\chi^{2}$-test. In order to adjust for multiple comparisons, a two-tailed probability level of 0.01 or less was considered to represent statistical significance.

\section{Results}

During the 11 year study period 40 triplet pregnancies were delivered after a gestational age of 20 weeks or more, and were matched with 80 twin pregnancies. The majority of the triplets $(82 \%)$ were a result of assisted reproduction, compared with $36 \%$ of the twin pregnancies $(P<0.001)$. The relative number of women referred to our hospital with triplet pregnancies $(40 \%)$ was twice that with twins $(21 \%)$. The main indication for referral in both groups was pre-term labor.

\subsection{Course of pregnancy}

A correct diagnosis of the number of fetuses was made in all patients at $10(5-29)$ weeks in triplet pregnancies and at $13(5-31)$ weeks gestation in twins. Cervical cerclage was not applied in twin pregnancies, but three women with triplets received a cerclage at the end of the first trimester because of suspected early cervical changes. In the triplet group, amniocentesis for antenatal diagnosis of fetal chromosomal abnormalities was performed on the basis of maternal age in four patients, and trisomy 21 was diagnosed in one fetus; the parents decided to accept the fetal abnormality and the pregnancy continued uneventfully. In the triplet group, one anencephalic fetus was diagnosed by ultrasound at a gestational age of 16 weeks. In the twin group, one fetus with a gastroschisis and, in another patient, a fetus with multicystic kidneys were diagnosed by ultrasound; the parents decided to accept these abnormalities.

Clinical characteristics of pregnancy and delivery in both groups are presented in Table 1. Most of the women with triplets $(85 \%)$ but only half the number of mothers with twins (49\%) were admitted to the hospital at some time during pregnancy, because of subjective complaints or maternal complications. The difference in antenatal complications was mainly due to the significantly higher incidence of pre-term labor requiring intravenous tocolysis in triplets as compared to twins $(P<0.01)$. The median duration of tocolytic treatment was $14(1-74)$ days in the triplet group and $5(1-55)$ days in twin pregnancies $(P<0.01)$. In the triplet group, 14 patients (35\%) and in the twin group 11 patients $(14 \%)$ received prophylactic steroids to enhance fetal lung maturation. The incidence of pregnancyinduced hypertension and preeclampsia was not different between triplet and twin pregnancies. Of the nine patients with triplets and pregnancy-induced hypertension, three cases were severe with diastolic blood pressures $>110 \mathrm{mmHg}$, compared with two of 10 cases in twins.

\subsection{Labor and delivery}

Of the $\mathbf{4 0}$ patients with triplets, 36 were delivered before a gestational age of 37 weeks (median 32 weeks), 
Table 1

Clinical characteristics of pregnancy and delivery in a study group of triplet pregnancies and a control group of twin gestations matched for maternal age and parity

\begin{tabular}{|c|c|c|}
\hline & $\begin{array}{l}\text { Triplets } \\
(n=40)\end{array}$ & $\begin{array}{l}\text { Twins } \\
(n=80)\end{array}$ \\
\hline Maternal age (years) & $31(21-39)$ & $31(21-39)$ \\
\hline \multicolumn{3}{|l|}{ Parity } \\
\hline 0 & 22 & 44 \\
\hline$\geq 1$ & 18 & 36 \\
\hline \multicolumn{3}{|l|}{ Pregnancy complications } \\
\hline Pre-term labor & $36(90 \%)$ & $48(60 \%)^{*}$ \\
\hline Pre-term rupture of membranes & $5(12 \%)$ & $19(24 \%)$ \\
\hline Pregnancy-induced hypertension & $9(22 \%)$ & $10(12 \%)$ \\
\hline Preeclampsia & $1(2 \%)$ & $3(4 \%)$ \\
\hline Gestational diabetes & $2(5 \%)$ & $5(6 \%)$ \\
\hline Anemia & $15(38 \%)$ & $22(28 \%)$ \\
\hline Antenatal hospitalization & $34(85 \%)$ & $39(49 \%)^{*}$ \\
\hline Gestational age (weeks) & $28(21-36)$ & $31(23-40)^{*}$ \\
\hline Duration (days) & $16(1-103)$ & $3(1-58)^{*}$ \\
\hline \multicolumn{3}{|l|}{ Delivery } \\
\hline Gestational age (weeks) & $32(23-37)$ & $35.5(23-41)^{*}$ \\
\hline $23-28$ & 11 & 9 \\
\hline 29-32 & 11 & 15 \\
\hline $33-36$ & 14 & 24 \\
\hline$\geq 37$ & 4 & $32^{*}$ \\
\hline \multicolumn{3}{|l|}{ Mode of delivery } \\
\hline Vaginal & 15 & $63^{*}$ \\
\hline Cesarean section & 25 & $17^{*}$ \\
\hline Postpartum hemorrhage & 6 & 5 \\
\hline
\end{tabular}

Values are given as median (range) or number (percentage), as appropriate.

$* P \leq 0.01$.

a significant difference $(P<0.001)$ with 48 of 80 patients in the twin group (median 35.5 weeks). There is also a marked difference in very pre-term birth before 29 weeks of gestation between triplets $(25 \%)$ and twins $(9 \%)$ $(P<0.01)$. The incidence of cesarean section was significantly higher in patients with triplets $(62 \%)$ than with twins $(21 \%)$. The indication was elective in $30 \%$ of triplets and in $12 \%$ of twin pregnancies $(P<0.001)$; the remaining indications were fetal distress and non-vertex presentation of the first twin. All cesarean sections were performed by low transverse incision.

Of the placentae in the triplet group, $30(75 \%)$ were triamniotic-trichorionic, five $(12.5 \%)$ triamnioticbichorionic and three $(7.5 \%)$ were triamniotic-monochorionic. One of the three monochorionic placentae was associated with intrauterine death of one fetus. Sixty $(75 \%)$ of the twin placentae were biamnioticbichorionic, $12(15 \%)$ were biamniotic-monochorionic and one was monoamniotic. Of two triplet and seven twin placentae no complete data were available.

\subsection{Neonatal outcome}

Data on neonatal outcome are summarized in Table
2. There were 12 fetal losses among the 120 triplets, two with a weight of less than $500 \mathrm{~g}$. Among the 108 liveborn infants there were 18 neonatal deaths. Corrected for three infants delivered at less than 24 weeks gestation with a birth-weight range of $400-480 \mathrm{~g}$ and one infant with anencephaly, the perinatal mortality of triplet pregnancies was $20 \%$; with $8.9 \%$, the perinatal mortality in twins, corrected for lethal congenital malformations and a birth-weight of less than $500 \mathrm{~g}$, was significantly less $(P<0.01)$. The difference is mainly caused by the stillbirth rate of $8.5 \%$ in triplets compared with $2.5 \%$ in twins $(P<0.01)$. In three triplet pregnancies and in four twin pregnancies all fetuses were lost. Neonatal mortality was associated with very pre-term birth $(<29$ weeks) in $44 \%$ of cases in triplets and in $38 \%$ in twins, an insignificant difference.

Triplets had a significantly lower median birth-weight than twins, but there is no significant difference in birthweight index between groups. The median hospital stay was significantly longer in the triplets, mainly related to

Table 2

Perinatal outcome in triplet pregnancies and in twin gestations matched for maternal age and parity

\begin{tabular}{|c|c|c|}
\hline & $\begin{array}{l}\text { Triplet births } \\
(n=120)\end{array}$ & $\begin{array}{l}\text { Twin births } \\
(n=160)\end{array}$ \\
\hline Live births & $108(91.5 \%)$ & $156(97.5 \%)^{*}$ \\
\hline Birth-weight (g) & $1478(300-2980)$ & $2030(300-3650)^{*}$ \\
\hline Birth-weight index & $0.84(0.10-1.14)$ & $0.86(0.17-1.32)$ \\
\hline Stillbirths ${ }^{\mathrm{a}}$ & $10(8.5 \%)$ & $3(2.5 \%)^{*}$ \\
\hline Birth-weight (g) & $680(510-970)$ & $720(520-900)$ \\
\hline 5 min Apgar-score $<7$ & $36(33 \%)$ & $17(11 \%)^{*}$ \\
\hline Early neonatal deaths & $10(10.2 \%)$ & $7(4.5 \%)$ \\
\hline Birth-weight (g) & $870(650-1130)$ & $830(600-1020)$ \\
\hline Late neonatal deaths & $4(4.1 \%)$ & $4(2.7 \%)$ \\
\hline Birth-weight (g) & $1120(970-1555)$ & $900(780-1030)$ \\
\hline Perinatal mortality ${ }^{\mathbf{a}}$ & $24(20 \%)$ & $14(8.9 \%)^{*}$ \\
\hline \multicolumn{3}{|l|}{ Pregnancies with discharge } \\
\hline \multicolumn{3}{|l|}{ Neonatal complications } \\
\hline Hospital stay (days) & $30(1-317)$ & $13(1-143)^{*}$ \\
\hline Congenital anomalies & 6 & 7 \\
\hline Episodes of bradycardia & 35 & 37 \\
\hline Ventilatory support & 37 & 43 \\
\hline Seizures & 2 & 2 \\
\hline Necrotizing enterocolitis & 2 & 2 \\
\hline Hyperbilirubinemia & 54 & $51^{*}$ \\
\hline Intraventricular hemorrhage & 2 & 6 \\
\hline $\begin{array}{l}\text { Patent ductus arteriosus } \\
\text { Bronchopulmonary }\end{array}$ & 5 & 5 \\
\hline dysplasia & 4 & 4 \\
\hline
\end{tabular}

Values are given as median (range) or number (percentage), as appropriate.

${ }^{*} P<0.01$. ${ }^{a}$ Birth-weight $>500 \mathrm{~g}$ and corrected for lethal congenital malformations. 
the lower birth-weight. Except for hyperbilirubinemia, which was observed more frequently in triplets, there were no significant differences between triplets and twins in the incidence of major neonatal complications. The number of infants needing ventilatory support was not different between triplets and twins. Triplets received ventilatory support during a median period of 4 (1-52) days compared to $2(1-59)$ days in twins, an insignificant difference. In the triplet group, intraventricular hemorrhage grade II was diagnosed in a 25 week infant, and a grade IV hemorrhage in a 27 weeks infant; both babies died. In twins, intraventricular hemorrhage grade I-II occurred in five infants, grade III in one; the six infants were born between 25 and 30 weeks gestation, and four of them died. Of the triplets, four infants had a club foot without other congenital abnormalities; one infant of a 36 year-old mother had Down's syndrome and one infant was anencephalic, both diagnoses were made antenatally. In the twin group, one infant had prune belly syndrome, one was born with gastroschisis and one infant had an aorta stenosis. Two infants were born with renal dysplasia, one of which was diagnosed antenatally by ultrasound; hypospadia was observed in two neonates.

\section{Discussion}

The majority of the triplet pregnancies in our study were a result of infertility treatment. Consideration of the clinical options of reduction to twins and a policy of expectant management must take into account the risks of maternal, fetal and neonatal complications and the probability of taking home at least one live and healthy infant associated with both approaches. Our study of triplet and twin pregnancies shows that pre-term labor is the most significant antenatal problem, in particular in triplet gestations, with no significant differences between both types of multifetal pregnancy with regard to other major complications. Pregnancy-induced hypertensive disorders are considered a major complication of higher order multifetal pregnancy [17]. Indeed, pregnancy-induced hypertensive disorders occurred in $22 \%$ of our triplet pregnancies, in accordance with an incidence of $13 \%-20 \%$ reported by others [10-13]. However, the majority of patients had mild non-proteinuric hypertension, and no difference between the incidence of preeclampsia in triplet and twin gestation could be demonstrated.

Pre-term delivery is the most important determinant of neonatal outcome in multifetal gestation. The incidence of delivery before 37 completed weeks in triplet gestation ranges from $64 \%$ to almost $100 \%$ in various studies $[1,6,7,10-16]$, compared with $90 \%$ in our study. The median gestational age at delivery in triplet pregnancy was 32 weeks in our study, compared with 32-34 weeks in other reports $[1,6,10-16]$. Our study may be biased towards lower gestational ages at delivery due to the high referral rates because of pre-term labor, in particular in the triplet group. However, our results are similar to those of a recently reported large study of 198 triplet pregnancies of which $95 \%$ were delivered at less than 37 weeks, with an average gestational age at delivery of 34 weeks [15]. In contrast to that study, in which ambulatory perinatal nursing and home uterine contraction monitoring were uniformly used, we applied standard antenatal care on an outpatient basis as long as the course of pregnancy remained uncomplicated. A recent review failed to provide evidence of a significant contribution of the routine use of antepartum hospitalization or prophylactic tocolytic treatment to the prognosis of triplet pregnancies [15].

The optimal management of delivery for triplets is debated. In their review, Petrikovsky and Vintzileos [17] show that the vaginal route was primarily chosen as the mode of delivery in most reports between 1978 and 1985 , with cesarean section rates in the larger series varying between $7 \%$ and $32 \%$. However, more recent reports indicate a dramatic increase in the number of cesarean sections in triplet as well as in twin gestations, reaching levels of $80 \%-100 \%[6,15,16]$. Although some authors suggest improved neonatal outcome in association with cesarean delivery [15], there are no randomized trials to support this view.

In our study the stillbirth as well as the total perinatal mortality rates were significantly higher in triplets than in twins. Comparison of perinatal mortality rates between studies may be misleading as the definition varies from report to report. In general, the available recent literature on the outcome of triplet pregnancies indicates a perinatal mortality rate ranging from $13.3 \%-33 \%$ $[10-13,17]$ with some notable exceptions of mortality rates between $4 \%-10 \%[6,15,16]$. Our findings are in close agreement with those in a recent large study showing a $16 \%$ perinatal mortality in triplet pregnancies, while $15 \%$ of the liveborn infants did not survive infancy [18]. For comparison with the results of selective fetal reduction it is more important to consider the relative number of pregnancies terminating in at least one live infant than the overall perinatal mortality. In our study, $92.5 \%$ of the mothers with triplet gestation had at least one surviving infant, compared with $95 \%$ with twin pregnancy. In comparison, discharge home with at least one infant was $88.2 \%$ in a recent study of 34 triplet pregnancies with reduction to twins [7].

Our study confirms that triplets have a lower median birth-weight and gestational age at delivery, and a longer neonatal stay in hospital than twins. However, we found no significant differences in major neonatal complications between triplets and twins.

Recent data on reduction of triplets to twins indicate a loss of the entire pregnancy of approximately $8 \%-16 \%$ to 24 weeks $[7,19]$ and the experience of the operator 
rather than the method used appears to be the key determinant of success for an individual patient [19]. Although reduction of triplet to twin pregnancy may be expected to reduce perinatal mortality after 24 weeks, the results of our study do not show significant differences between maternal morbidity and neonatal mortality and morbidity in triplet and twin pregnancies managed expectantly, and no marked effect of fetal reduction on these outcomes is to be expected. Also, in the only study in which the outcome of triplet gestations managed expectantly was compared with that in triplet pregnancies reduced to twins, no significant effect on neonatal mortality was demonstrated [7].

The data obtained in our study may be used to counsel women with a triplet pregnancy considering selective reduction to twins with regard to the anticipated perinatal outcome of expectantly-managed triplet and twin pregnancies. However, the decision to continue pregnancy or to opt for fetal reduction does not only depend on obstetric considerations but also on the socioeconomic consequences of multiple pregnancy, on the complex ethical problems of fetal reduction, and on the emotional and psychologic problems associated with both policies [20]. Since the majority of triplet and higher-order multifetal pregnancies are a result of infertility treatment, we feel that all methods of assisted reproduction should aim at prevention of multifetal gestation. We share the distress expressed by others [19] about the recently advocated extremely aggressive application of assisted reproductive techniques with incorporation of multifetal pregnancy reduction as an adjunct of treatment [21].

\section{References}

[1] Levene MI, Wild J, Steer P. Higher multiple births and the modern management of infertility in Britain. Br J Obstet Gynaecol 1992; 99: 607-13.

[2] Berkowitz RL, Lynch L, Chitkare U. Selective reduction of multifetal pregnancies in the first trimester. N Engl J Med 1988; 318(16): 1043-7.

[3] MacLennan AH. Multiple gestations, Maternal Fetal Medicine: Principles and Practice. Creasy RK, Resnik R, eds. Philadelphia, W.B. Saunders 1984, pp. 527-38.
[4] Editorial. Selective fetal reduction. Lancet 1988; 2: 773-5.

[5] Wapner RJ, Davis GH, Johnson A et al. Selective reduction of multifetal pregnancies. Lancet 1990; 1 : 90.

[6] Sassoon DA, Castro C, Davis JL, Hobel CJ. Perinatal outcome in triplet versus twin gestation. Obstet Gynecol 1990; 75: 817-20.

[7] Lipitz S, Reichman B, Uval J et al. A prospective comparison of the outcome of triplet pregnancies managed expectantly or by multifetal reduction to twins. Am J Obstet Gynecol 1994; 170: 874-9.

[8] Davey DA, MacGillivray I. The classification and definition of the hypertensive disorders of pregnancy. Am J Obstet Gynecol 1988; 158: 892-8.

[9] Kloosterman GJ. On intrauterine growth. Int J Gynaecol Obstet 1970; 8: 895-912.

[10] Syrop CH, Varner MW. Triplet gestation: maternal and neonatal implications. Acta Genet Med Gemellol 1985; 34: 81-8.

[11] Itzkowic D. A survey of 56 triplet pregnancies. Br J Obstet Gynaecol 1979; 86: 23-8.

[12] Daw E. Triplet pregnancy. Br J Obstet Gynecol 1978; 85: 505-9.

[13] Michlewitz H, Kennedy J, Kawada C, Kennison R. Triplet pregnancies. J Reprod Med 1981; 26: 243-6.

[14] Gonen R, Heyman E, Asztalos EV, Ohlsson A, Pitson LC, Shennan AT, Milligan JE. The outcome of triplet, quadruplet and quintuplet pregnancies managed in a perinatal unit: Obstetric, neonatal, and follow-up data. Am J Obstet Gynecol 1990; 162: 454-9.

[15] Newman RB, Hamer C, Miller MC. Outpatient triplet management: A contemporary review. Am J Obstet Gynecol 1989; 161: 547-55.

[16] Boulot P, Hedon B, Pelliccia G et al. Favourable outcome in 33 triplet pregnancies managed between 1985-1990. Eur J Obstet Gynecol Reprod Biol 1992; 43: 123-9

[17] Petrikovsky BM, Vintzileos AM. Management and outcome of multiple pregnancy of high fetal order: Literature Review. Obstet Gynecol Survey 1989; 44: 578-84.

[18] Botting BJ, MacDonald Davies I, MacFarlane AJ. Recent trends in the incidence of multiple births and associated mortality. Arch Dis Child 1987; 62: 941-50.

[19] Evans MI, Dommergues M, Timor-Tritsch I et al. Transabdominal versus transcervical and transvaginal multifetal pregnancy reduction: International collaborative experience of more than one thousand cases. Am J Obstet Gynecol 1994; 170: 902-9.

[20] Garel M, Blondel B. Assessment at 1 year of the psychological consequences of having triplets. Hum Reprod 1992; 7: 729-32.

[21] Ayers JWT, Petersen EP, Knight L, Peterson S. Incorporation of transvaginal embryo reduction (TVER) with an agressive IVF/GIFT/ZIFT program to optimize pregnancy outcome. Fertil Steril 1991; 56: 5173. 